\title{
Physiology of Connective Tissues and Fascia Update Knowledge for Orthopaedic Surgeons \& Rehabilitation Carers
}

\author{
Ping-Chung LEUNG ${ }^{*}$ \\ ${ }^{1}$ Professor Emeritus, Department of Orthopaedics, The Chinese University of Hong Kong, Hong Kong
}

${ }^{*}$ Corresponding author: Ping-Chung LEUNG, 5/F School of Public Health Building, Prince of Wales Hospital, Shatin, Hong Kong; Tel: 8522252 8868; Fax: 8522632 5441; Email: pingcleung@cuhk.edu.hk

Received: April 04, 2019; Accepted: April 16, 2019; Published: April 19, 2019;

\begin{abstract}
Unlike traditional views, fascia and connective tissues are not just unimportant structure without special function. Instead, fascia can be considered a large organ without boundaries, existing as a wide-spread network, carrying multiple function. In musculoskeletal functions, fascia supports joint mobilizations, helping to form architecture of stabilizing and motivating complex controlling joint stabilities and movements. Although fascia does carry a simple function of providing tissue protection and gliding, it fulfils this role via active channels. Fibrocytes contained in the fascia are actively responding to stress and tension; while stem cells are supporting tissue regeneration.

Proprioceptive receptors in the fascia carry messages to the brain central which respond quickly and actively to stabilization requirements and other activities. The interstitial fluid within the fascia and neighboring cells and muscles are not simple "leakages" from vascular and lymphatic channels. Instead, the cellular activities within the fascia which helps to form the architecture of bodily movements, could be an important influencing force of both vascular and lymphatic circulation. The interstitial fluid, therefore, deserves to be considered the third fluid system of the human body with its specific function. The new discoveries of the fascia, therefore, carry special implications on physiological and pathological changes relevant to therapy and rehabilitation.
\end{abstract}

\section{Classical view of Connective Tissues}

Since anatomical dissections started in quest for better understanding of the human body, connective tissues existing outside and surrounding important structures and organs are revealed and described. The general attitude of anatomists and later surgeons (who dissect and give constructive measures to tissues and organs) towards connective tissues is that they are simple separating sheets of life tissues without specific function. Subsequent, observations have made a variety of fibrous connective tissues which have been given specific terminologies like: aponeurosis, ligament, tendon, retinaculum, joint capsule, epineurium, meninges, vessel coverages and periosteum. All these structures belong to simple interconnecting tensional tissues, and many varieties have been called "fascia" which in Latin, refers to bound together straps and bundles. Since fascia and other connective tissues are simple separation structures carrying no specific function, destruction and disturbance of which is expected to be followed by spontaneous replacement of a thicker and more fibrotic tissues.

\section{Reveal of the Complicated Function of Connective Tissues and Fascia}

\section{a) Musculoskeletal Function}

When joint motions were studied in details, biomechanical observations revealed that although muscle contraction is the main motivating force, contractile tissues around and special fascial arrangements all participate in synchronous supportive contractile or relaxant activities. It is now clear that connective tissues carry two district functions: to allow structural gliding and to facilitate transfer of forces. Tissue gliding is important for functional protection. The fact that important structures like muscles and organs always possess two layers of soft tissue coverages (fascia), one inner sheet wrapping tight the structure and one outside sheet to facilitate gliding. In the case of muscles and joints, the surrounding fascia ensures that the related joint remains stable in whatever position change [1].

The classical observation that fascia helps to hold small vessels and nerves which need to provide nutrition and messages in their role of interconnection. The real situation is that not only does the fascia provide passages for the vascular and nerve channels but nerve receptors: muscle spindles; Golgi tendon organs and Ruffini corpuscles; laminated or paciniform corpuscles; and free nerve endings are scattered around [1]. The implication is that movements affecting the fascia would be simultaneously initiating neurological messages up and down the fascia, possibly also extending to the connecting network of connective tissues [2]. The Myofascial connections between the adjacent muscle groups would allow much dynamic planning for therapeutic measures designed for musculoskeletal disorders [3].

\section{b) Fascial Cytology}

Cells in the fascial connective tissues carry specialized functions. There are two main types: fibrocytes and stem cells. The fibrocytes are 
provided with integrin receptors on their surfaces and are mechanically coupled to the actin cytoskeleton of the cell, forming a pathway to sense external forces and allow the cell to respond with changes of cell shape. The cells can activate internal chemical signaling pathways, increase stress-fiber assembly and adhesive strength, and form focal adhesions in response to externally applied mechanical forces [4].

The dynamic properties of the fibrocytes help with muscular activities suitable for different forms of stress and requirements. They are also responsible for the cyclin changes of intramuscular pressure which assists in venous return of blood to the heart. The presence of nerve fibrils and sensors within the fascia network has granted the fascia a specific functional role. Mechanoreceptors, also called muscle receptors are arranged in the context of force transmissions i.e. around and within muscle and connective tissues. Other receptors for proprioception are concentrated in those areas where tensile stresses are mostly felt and they are responsible for the maintenance of joint integrity and stability. Since the fascial system all over the body overlooks the proprioceptive stability of the whole body, the role of the connective tissues as an important proprioception stabilizer in the locomotor apparatus, individually and in collaboration with the Central Nervous System cannot be overstressed [1].

Another type of cells in the soft tissues of the fascia is the stem cells that possess regenerative power obviously responsible for the reparative need of musculoskeletal tissues. The dynamic nature of the stem cells: their versatility of regenerative direction and their ability to freely move following the network of fascial arrangement is well known but yet has not been thoroughly studied. Not only are the mechanisms of cellular transformation to various deficiency or reparative needs important, but the mobility of the cells is crucial. The relationship between inflammation and cellular degradation occurring within the soft tissues would be of major concern.

\section{c) Fluid Flow}

The proper function of mechanical stability and neural transmission demands an efficient fluid flow within the fascial network. Likewise, the same dynamic flow would allow efficient movement of stem cells. It has always been taken for granted that interstitial fluid occurs between cells, tissues and organs and that it passively serves the neighboring tissues and structures without additional role in the overall well-being of the system. The feeding of the interstitial fluid is also taken for granted that either the general vascular circulation or the lymphatic system would be taking care of this spatial fluid. It is of course true that the interstitial fluid comes from both the general circulation and the lymphatic system. But the discovery of neural sensors and special cells within the fascia and in the interstitial fluid itself has changed the old belief.

The general vascular circulation is responsible to maintain the normal fluid balance of the body while at the same time taking care of the lymphatic system. Venous return and lymphatic flow on the other hand are assisted by the "muscle pump" of the lower limb: a manifestation of programmed muscle contractions. Studies of the fascia and interstitial fluid revealed that cellular activities in the fascia are constantly regulating fluid flow and muscle contractures actively. It is therefore concluded that three systems of fluid flow are interconnecting and they are mutually influencing one another's functional flow efficiency in a harmonious balance. This balance is crucial for the transport of nutrients, passage of neurological messages and tissue repair [5].

\section{Significance of the New Findings}

The existence of the three circulating fluid systems that maintains a harmonious balance of activities in the musculoskeletal system gives special insight for the orthopaedic field. Surgeons during their surgical dissections would give special considerations to the fascia, particularly when it is related directly to ligaments, aponeurosis and tendons. Rehabilitation workers would be giving special attention to their routine stretches and manipulations to ensure that the effects would stimulate fascial stretches and movements so as to achieve effective stimulations to the nerve receptors and different types of cells in the soft tissue. Individuals aware of the special fascial physiology would start adopting a life style that ensures mobility and efficiency of fluid flow. The adaptation carries a comprehensive change of attitude not only for leisure training but also during work hours.

One scientific report was published in March 2018 describing in detail the fascial anatomy and the interstitial fluid and its contents. It was postulated that those structures could be important in cancer metastasis, edema, fibrosis and mechanical functioning of many tissues and organs [6]. Indeed, fascia exist not only in musculoskeletal structures, but everywhere in the human body. It exists in close harmony with its adjacent tissues and organs sharing their functional need, while at the same time help to fulfill the physiological role of the blood circulatory and lymphatic garaging systems.

\section{References}

1. Van den Wal J (2009) Architecture of Connective Tissue in the musculoskeletal system: Fascia Research II Elsevier, Munchen 2009: 21-27.

2. Goddard MD, Red ID (1965) Movements induced by straight leg raising in the lumbo-sacral roots, nerves and plexus, and in the intrapelvic section of the sciatic nerve. J Neurol Neurosurg Psychiatry 28: 12-18.

3. Huijing PA (2003) Muscular force transmission necessitates a multi-level integrative approach to the analysis of function of skeletal muscle. Exerc Sport Sci Rev 31: 167-175.

4. Grinnell F (2003) Fibroblast biology in three-dimensional collagen matrices. Trends Cell Biol 13: 264-269

5. Peter Chin Wan Fung, Regina Kit Chee Kong (2016) The Integrative Five-Fluid Circulation System in the Human Body. Open $J$ Molecular and Integrative Physiology 6: 45-97.

6. Benias PC, Wells RG, Theise ND (2018) Structure and Distribution of an unrecognized interstitium in Human Tissues. www.anature.com/scientific report.

\section{Citation:}

Ping-Chung L (2019) Physiology of Connective Tissues and Fascia Update Knowledge for Orthopaedic Surgeons \& Rehabilitation Carers. Integr $J$ Orthop Traumatol Volume 2 (1): 1-2. 